\title{
Theory of internal transitions of charged excitons in quantum wells in magnetic fields
}

\author{
A. B. Dzyubenko \\ Institut für Theoretische Physik, J.W. Goethe-Universität, 60054 Frankfurt, Germany \\ General Physics Institute, RAS, Moscow 117942, Russia
}

A. Yu. Sivachenko

The Weizmann Institute of Science, Rehovot 76100, Israel

(March 6, 2017)

For charged semiconductor complexes in magnetic fields $B$, we discuss an exact classification of states, which is based on magnetic translations. In this scheme, in addition to the total orbital angular momentum projection $M_{z}$ and electron and hole spins $S_{e}, S_{h}$, a new exact quantum number appears. This oscillator quantum number, $k$, is related physically to the center of the cyclotron motion of the complex as a whole. In the dipole approximation $k$ is strictly conserved in magnetooptical transitions. We discuss implications of this new exact selection rule for internal intraband magneto-optical transitions of charged excitons $X^{-}$in quantum wells in $B$.

73.20.Dx, 71.70.Di, 76.40.+b

Recently, there has been considerable experimental and theoretical interest in charged excitons $X^{-}$and $X^{+}$ in magnetic fields $B$ in $2 \mathrm{D}$ systems. Experimentally, magneto-optical interband [1] transitions of charged excitons have been studied extensively. Theoretically, the binding of charged excitons $X^{-}$has been considered in quantum dots [2], in a strictly-2D system in the highmagnetic field limit [3], and in realistic quantum wells at finite $B$ 仼. In all these theoretical works on charged excitons, the existing exact symmetry - magnetic translations - has not been identified. The aim of the present theoretical work is to describe in some detail this symmetry and its manifestations in intraband magneto-optical transitions (see also [0]). Experimental evidences for internal $X^{-}$singlet and triplet transitions have been reported very recently in [6], where also comparison with quantitative calculations is presented.

We consider a system of interacting particles of charges $e_{j}$ in a magnetic field $\mathbf{B}=(0,0, B)$ described by the Hamiltonian

$$
H=\sum_{j} \frac{\hat{\boldsymbol{\pi}}_{j}^{2}}{2 m_{j}}+\frac{1}{2} \sum_{i \neq j} U_{i j}\left(\mathbf{r}_{i}-\mathbf{r}_{j}\right),
$$

here $\hat{\boldsymbol{\pi}}_{j}=-i \hbar \boldsymbol{\nabla}_{j}-\frac{e_{j}}{c} \mathbf{A}\left(\mathbf{r}_{j}\right)$ is the kinematic momentum operator of the $j$-th particle in $\mathbf{B}$ and $U_{i j}$ are the potentials of interactions that can be rather arbitrary. Dynamical symmetries of (11) are the following. In the symmetric gauge $\mathbf{A}=\frac{1}{2} \mathbf{B} \times \mathbf{r}$, there is the axial symmetry about the $z$-axis $\left[H, \hat{L}_{z}\right]=0$, where $\hat{L}_{z}=\sum_{j}\left(\mathbf{r}_{j} \times-i \hbar \boldsymbol{\nabla}_{j}\right)_{z}$.
Therefore, the total angular momentum projection $M_{z}$, an eigenvalue of $\hat{L}_{z}$, is a good quantum number. In a uniform $\mathbf{B}$, the Hamiltonian (1) is also invariant under a group of magnetic translations whose generators are the components of the operator $\hat{\mathbf{K}}=\sum_{j} \hat{\mathbf{K}}_{j}$, where $\hat{\mathbf{K}}_{j}=\hat{\boldsymbol{\pi}}_{j}-\frac{e_{j}}{c} \mathbf{r}_{j} \times \mathbf{B}$ (see, e.g., [7]). $\hat{\mathbf{K}}$ is the exact integral of the motion: $[H, \hat{\mathbf{K}}]=0$. The components of $\hat{\mathbf{K}}$ and $\hat{\boldsymbol{\pi}}=\sum_{j} \hat{\boldsymbol{\pi}}_{j}$ commute in $\mathbf{B}$ as

$$
\left[\hat{K}_{x}, \hat{K}_{y}\right]=-\left[\hat{\pi}_{x}, \hat{\pi}_{y}\right]=-i \frac{\hbar B}{c} Q \quad, \quad Q \equiv \sum_{j} e_{j}
$$

while $\left[\hat{K}_{p}, \hat{\pi}_{q}\right]=0, p, q=x, y$. For neutral complexes (atoms, excitons, biexcitons) $Q=0$, and classification of states in $B$ are due to the continuous two-component vector - the $2 \mathrm{D}$ magnetic momentum $\mathbf{K}=\left(K_{x}, K_{y}\right)$. For charged systems the components of $\hat{\mathbf{K}}$ cannot be observed simultaneously. This determines the macroscopic Landau degeneracy of exact eigenstates of (1). For a dimensionless operator $\hat{\mathbf{k}}=\sqrt{c / 2 \hbar B|Q|} \hat{\mathbf{K}}$ we have $\left[\hat{k}_{x}, \hat{k}_{y}\right]=$ $-i Q /|Q|$. Therefore, $\hat{k}_{ \pm}=\left(\hat{k}_{x} \pm i \hat{k}_{y}\right) / \sqrt{2}$ are Bose raising and lowering ladder operators: $\left[\hat{k}_{+}, \hat{k}_{-}\right]=-Q /|Q|$. It follows then that $\hat{\mathbf{k}}^{2}=\hat{k}_{+} \hat{k}_{-}+\hat{k}_{-} \hat{k}_{+}$has discrete oscillator eigenvalues $2 k+1, k=0,1, \ldots$. Since $\left[\hat{\mathbf{k}}^{2}, H\right]=0$ and $\left[\hat{\mathbf{k}}^{2}, \hat{L}_{z}\right]=0$, the exact charged eigenstates of (1), in addition to the electron $S_{e}$ and hole $S_{h}$ spin quantum numbers, can be simultaneously labeled by the discrete quantum numbers $k$ and $M_{z}$. The labelling therefore is $\left|k M_{z} S_{e} S_{h} \nu\right\rangle$. Here $\nu$ is the "principal" quantum number, which can be discrete (bound states) or continuous (unbound states forming a continuum) 河. The $k=0$ states are Parent States (PS's) within a degenerate manifold. All other Daughter states in each $\nu$-th family are generated out of the PS iteratively: for $Q<0$ $\left|k, M_{z}-k, S_{e} S_{h} \nu\right\rangle=\left(\hat{k}_{-}\right)^{k}\left|0, M_{z}, S_{e} S_{h} \nu\right\rangle / \sqrt{k !}$.

Let us discuss now magneto-optical transitions of charged complexes. In the dipole approximation the photon momentum is negligibly small. Therefore, the quantum number $k$ should be conserved in intra- and interband magneto-optical transitions. For interband transitions in a translationally-invariant system with a simple valence band this leads to a striking result [5] that the ground triplet $X_{t}^{-}\left(S_{e}=1\right)$ state is dark in photolumines- 


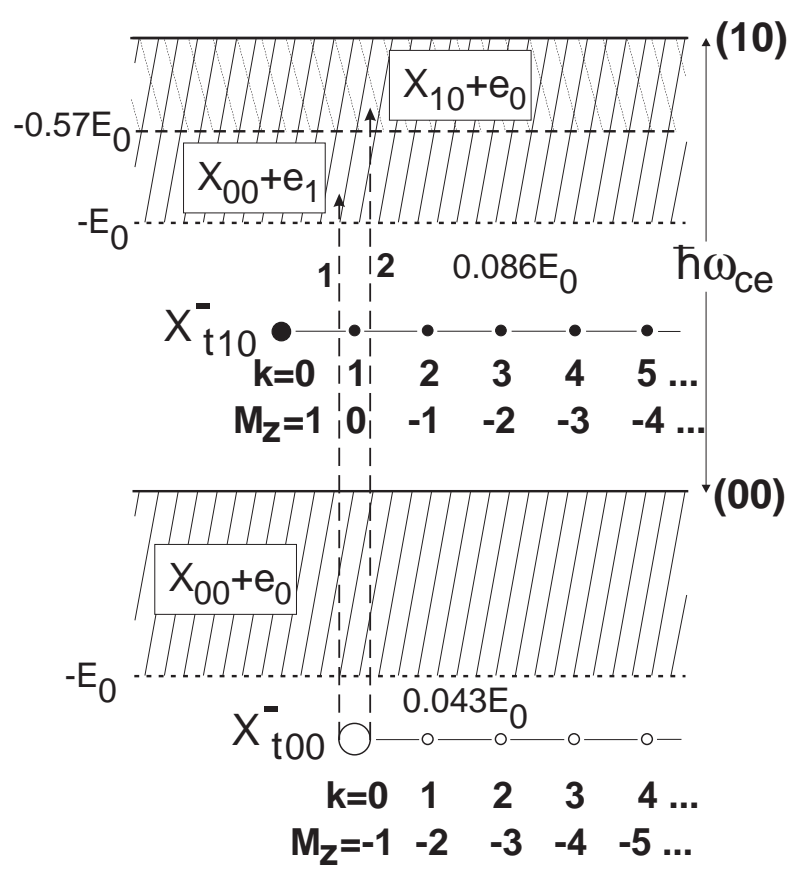

FIG. 1. Schematic drawing of bound and scattering electron triplet $2 e-h$ states in the lowest LL's $\left(N_{e} N_{h}\right)=(00),(10)$. The states are labeled by the total angular momentum projection $M_{z}$ and oscillator quantum number $k$. Separation between the $2 \mathrm{D} e$ - and $h$-layers $d=0$ (see inset to Fig. 2). The energy $E_{0}=\sqrt{\pi / 2} e^{2} / \epsilon l_{B}$ parametrizes the $2 \mathrm{D}$ system in the limit of high $B$. Large (small) dots correspond to the bound parent $k=0$ (daughter $k=1,2, \ldots$ ) $X^{-}$states; see text for further explanations.

cence at finite magnetic fields $B$ and in a quasi-2D system with a possible $e-h$ asymmetry (cf. [3]). Also, conservation of $k$ prohibits shake-up processes of an isolated singlet $X_{s}^{-}\left(S_{e}=0\right)$ state in $B$. In this work we concentrate on internal intraband transitions of charged complexes in a magnetic field. In these transitions conservation of $k$ follows from the commutativity $\left[\hat{V}^{ \pm}, \hat{\mathbf{K}}\right]=0$, where $\hat{V}^{ \pm} \sim \sum_{j} e_{j} \pi_{j}^{ \pm} / m_{j}$, is the Hamiltonian of the interaction with the radiation of polarization $\sigma^{ \pm}$and $\pi_{j}^{ \pm}=\pi_{j x} \pm i \pi_{j y}$. Other selection rules in this case are conservation of spins $S_{e}, S_{h}$ and $\Delta M_{z}= \pm 1$ in the $\sigma^{ \pm}$ polarization for the envelope function. Conservation of $k$ constitutes a new exact selection rule. It leads, in particular, to some striking consequences for bound-to-bound transitions from the triplet $X_{t}$ ground state.

To show this we shall consider the system of strictly$2 \mathrm{D} e$ - and $h$-layers separated by a distance $d$ (see inset to Fig. 2) in the limit of high magnetic fields. To understand internal $X^{-}$transitions, it is necessary to consider the eigenstates associated with higher Landau levels (LL's). To this end we use expansion in free LL's, which has been described in some detail elsewhere [8,5]. The calculated three-particle $2 e-h$ eigenspectra (electrons in the triplet

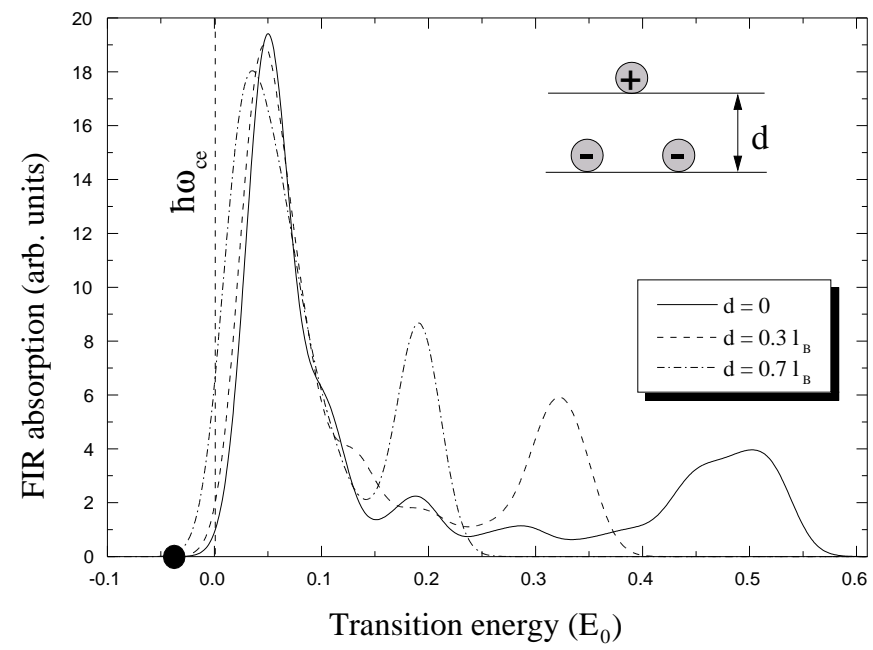

FIG. 2. Energies and dipole matrix elements of the inter-LL transitions from the ground $X_{t 00}^{-}$state in the high-field limit. Shown are the cases of three different separations $d=0,0.3 l_{B}$, and $0.7 l_{B}$ between the strictly-2D $e$ - and $h$-layers (see inset). A filled dot shows the position of the forbidden bound-to-bound $X_{t 00}^{-} \rightarrow X_{t 10}^{-}$transition for $d=0$.

state) in the two lowest LL's are shown for $d=0$ in Fig. 1. Generally, the eigenspectra associated with each LL consist of bands of finite width $\sim E_{0}=\sqrt{\pi / 2} e^{2} / \epsilon l_{B}$, where $l_{B}=(\hbar c / e B)^{1 / 2}$. The states within each such band form a continuum corresponding to the extended motion of a neutral magnetoexciton (MX) as a whole with the second electron in a scattering state. As an example, the continuum in the lowest $\left(N_{e} N_{h}\right)=(00) \mathrm{LL}$ consists of the MX band of width $E_{0}$ extending down in energy from the free (00) LL. This corresponds to the $1 s \operatorname{MX}\left(N_{e}=N_{h}=0\right)$ [9] plus a scattered electron in the zero LL, labeled $X_{00}+e_{0}$. The structure of the continuum in the $\left(N_{e} N_{h}\right)=(10)$ LL is more complicated: in addition to the $X_{00}+e_{1}$ band of the width $E_{0}$, there is another MX band of width $0.574 E_{0}$ also extending down in energy from the free $\left(N_{e} N_{h}\right)=(10)$ LL. This corresponds to the $2 p^{+}$exciton $\left(N_{e}=1, N_{h}=0\right)$ [9] plus a scattered electron in the $N_{e}=0 \mathrm{LL}$, labeled $X_{10}+e_{0}$. There are also bands (not shown in Fig. 1) above each free LL originating from the bound internal motion of two electrons in the absence of a hole [5]. Internal transitions to such bands have extremely small oscillator strengths and not discussed here. Bound $X^{-}$states (finite internal motions of all three particles) lie outside the continua (Fig. 1). In the limit of high $B$ the only bound $X^{-}$state in the zeroth LL $\left(N_{e} N_{h}\right)=(00)$ is the $X^{-}$-triplet. There are no bound $X^{-}$-singlet states [3, 1 in in contrast to the $B=0$ case. The $X^{-}$-triplet binding energy in zero LL's $\left(N_{e} N_{h}\right)=(00)$ is $0.043 E_{0}$ [3, , In the next electron LL $\left(N_{e} N_{h}\right)=(10)$ there are no bound $X^{-}$-singlets, and only one bound triplet state $X_{t 10}^{-}$, lying below the lower edge 
of the MX band [5]. The $X_{t 10}^{-}$binding energy is $0.086 E_{0}$, twice that of the $X_{t 00}^{-}$, and similar to the stronger binding of the $D^{-}$-triplet in the $N_{e}=1 \mathrm{LL}$ [8] .

We focus here on internal transitions in the $\sigma^{+}$polarization governed by the usual selection rules: spin conserved, $\Delta M_{z}=1$. In this case the $e$-CR-like inter-LL $\left(\Delta N_{e}=1\right)$ transitions are strong and gain strength with $B$. Both bound-to-bound $X_{t 00}^{-} \rightarrow X_{t 10}^{-}$and photoionizing $X^{-}$transitions are possible. For the latter the final three-particle states in the (10) LL belong to the continuum (Fig. 1), and calculations show that the FIR absorption spectra reflect its rich structure [5]. Transitions to the $X_{00}+e_{1}$ continuum are dominated by a sharp onset at the edge (transition 1) at an energy $\hbar \omega_{c e}$ plus the $X_{t 00}^{-}$binding energy. In addition, there is a broader and weaker peak corresponding to the transition to the $X_{01}+e_{0} \mathrm{MX}$ band, transition 2. The latter may be thought of as the $1 s \rightarrow 2 p^{+}$internal transition of the MX [10], which is shifted and broadened by the presence of the second electron. In accordance with this picture, it is visible from Fig. 2 that with increasing separation $d$ between the $e$ - and $h$-layers (when the exciton binding and, thus, transition energies are reduced and the $X-e$ interaction is effectively diminished), the second peak is redshifted and sharpened. Thus the $X^{-}$triplet behaves physically in the photoionizing boundto-continuum transitions as an exciton that very loosely binds an electron, and the two "parts" of the complex can absorb the FIR photon, to some extent, independently. The double-peak structure of the bound-to-continuum transitions is a generic feature for transitions from both the singlet and triplet ground $X^{-}$states in quasi-2D systems in strong $B$. Such transitions in translationally invariant systems are discussed theoretically in [6], where also experimental results for bound-to-continuum transitions are reported and comparison between theory and experiment is made.

The inter-LL bound-to-bound transition, $X_{t 00}^{-} \rightarrow X_{t 10}^{-}$, has a very specific spectral position: since the final state is more stronger bound, it lies below the e-CR energy $\hbar \omega_{c e}=\hbar e B / m_{e} c$. However, it has exactly zero oscillator strength, a manifestation of the magnetic translational invariance: the two selection rules - conservation of $k$ and $\Delta M_{z}=1$ cannot be satisfied simultaneously. Indeed, e.g., the $X_{t 00}$ PS (with $k=0$ ) has $M_{z}=-1$, while the $X_{t 10}$ PS has $M_{z}=1$, so that the usual selection rule $\Delta M_{z}=1$ cannot be satisfied. Localization of charged excitons breaks translational invariance and relaxes the $k$-conservation rule. As a result, the boundto-bound $X_{t 00}^{-} \rightarrow X_{t 10}^{-}$transition, which is prohibited in translationally-invariant systems, develops below the $e$ CR [5. Such a peak is a tell-tale mark of localization of charged triplet excitons.

In conclusion, we have studied the exact symmetry - magnetic translations - for charged excitons in $B$ and established its consequences for intraband magneto- optical transitions. In particular, we have shown that in translationally invariant quasi-2D system with a simple valence band the bound-to-bound transition from the triplet ground state $X_{t}^{-}$to the next electron Landau level is prohibited. In the presence of translationally-breaking effects (disorder, impurities etc.) the intraband boundto-bound triplet transition develops below the electron cyclotron resonance. This suggests a method of studying localization of charged excitons.

$\mathrm{ABD}$ is grateful to the Alexander von Humboldt Foundation for research support.

[1] See, e.g., K. Kheng, R.T. Cox, Merle Y. d'Aubigné et al. Phys. Rev. Lett. 71, 1752 (1993); G. Finkelstein, H. Shtrikman, and I. Bar-Joseph, Phys. Rev. Lett. 74, 976 (1995); A.J. Shields, M. Pepper, M.Y. Simmons, and D.A. Ritchie, Phys. Rev. B 52, 7841 (1995); D. Gekhtman, E. Cohen, Arza Ron, and L.N. Pfeiffer, Phys. Rev. B 54, 10320 (1996), D.R. Yakovlev, V.P. Kochereshko, R. Suris et al. Phys. Rev. Lett. 79, 3974 (1997) and references therein.

[2] A. Wojs and P. Hawrylak, Phys. Rev. B 51, 10880 (1995).

[3] J.J. Palacios, D. Yoshioka, and A.H. MacDonald, Phys. Rev. B 54, R2296 (1996).

[4] D.M. Whittaker and A.J. Shields, Phys. Rev. B 56, 15185 (1997).

[5] A.B. Dzyubenko and A.Yu. Sivachenko, condmat/9902086 cond-mat/9908406, to be published in Physica E.

[6] A.B. Dzyubenko, A.Yu. Sivachenko, H.A. Nickel, T.M. Yeo, G. Kioseoglou, B.D. McCombe, and A. Petrou, cond-mat/9907124, to be published in Physica E.

[7] J.E. Avron, I.W. Herbst, and B. Simon, Annals of Phys. 114, 431 (1978).

[8] A.B. Dzyubenko, Phys. Lett. A 165, 357 (1992); 173, 311 (1993).

[9] I.V. Lerner and Yu.E. Lozovik, Sov. Phys. JETP 51, 588 (1980).

[10] A.B. Dzyubenko, Pis'ma ZhETF 66, 588 (1997) [JETP Lett. 66, 617 (1997)]. 